\title{
Oral treatment with HE3286 ameliorates disease in rodent models of rheumatoid arthritis
}

\author{
DOMINICK L. AUCI ${ }^{1}$, KATIA MANGANO ${ }^{2}$, DANIEL DESTICHE ${ }^{1}$, STEVEN K. WHITE $^{1}$, YUJIN HUANG ${ }^{1}$, \\ DAVID BOYLE ${ }^{3}$, JAMES FRINCKE ${ }^{1}$, CHRISTOPHER L. READING ${ }^{1}$ and FERDINANDO NICOLETTI ${ }^{2}$ \\ ${ }^{1}$ HollisEden Pharmaceuticals, 4435 Eastgate Mall, Suite 400, San Diego, CA, USA; ${ }^{2}$ Department of Biomedical \\ Sciences, School of Medicine, University of Catania, Catania, Italy; ${ }^{3}$ Division of Rheumatology, Allergy \\ and Immunology, University of California at San Diego School of Medicine, La Jolla, CA, USA
}

Received October 30, 2009; Accepted December 9, 2009

\section{DOI: $10.3892 /$ ijmm_00000385}

\begin{abstract}
HE3286 (17 $\alpha$-ethynyl-5-androstene-3ß, 7ß, 17ß-triol) is an orally bio-available synthetic derivative of naturally occurring androstene- $3 \beta, 7 \beta, 17 \beta$-triol. Our present data show that oral treatment with HE3286, favourably influenced the course of arthritis in the rat model of adjuvant-induced arthritis (reduced cumulative disease scores and paw edema), and in the mouse model of collagen antibody-induced arthritis (reduced clinical paw scores). Importantly, HE3286 was not immune suppressive in human mixed lymphocyte reaction or in animals challenged with Coxsackie B3 virus. HE3286 is currently in phase I/II clinical trials in rheumatoid arthritis and ulcerative colitis and these findings further strengthen the possibility that HE3286 may represent an effective antiinflammatory agent useful for treating chronic inflammation with a more attractive safety profile than glucocorticoids or cyclooxygenase inhibitors.
\end{abstract}

\section{Introduction}

Rheumatoid arthritis (RA) is a systemic immune mediated inflammatory disease (IMID) that possesses a multifactorial pathogenetic pathway that includes dysregulated function of $\mathrm{T}$ cells, B cells, dendritic cells and macrophages that may result in an unbalanced network of endogenous chemokines and cytokines such as IL-1 and TNF- $\alpha$ (1). This forms the basic rationale for the broad use of newly developed biologics that selectively target the action of B cells (rituzimab) or endogenous TNF- $\alpha$ (infliximab, etanercept, rituximab, abatacept), in this disease. While generally efficacious (2-4), these agents are costly, cannot be administered orally (5) and are ineffective or contraindicated in certain patient

Correspondence to: Professor Ferdinando Nicoletti, Department of Biomedical Sciences, Via Androne, 83, 95124 Catania, Italy E-mail: ferdinic@unict.it

Key words: androstenetriol, autoimmune diseases, dehydroepiandrosterone, HE3286, immunotherapy, rheumatoid arthritis populations. Similar to glucocorticoids (6), they block the inductive phase of inflammation, are powerfully immune suppressive, and prolonged usage has been associated with adverse events (7). These also include reactivation of JC virus (8), a higher risk of developing other autoimmune pathologies such as psoriasis (9), lichen planus (10), lupus (11) and demyelinating neuropathies (12). TNF- $\alpha$ inhibitors may also increase the risk of reactivating viral latent infection with herpes zoster (13) or Epstein-Barr virus (14) and augments the risk of tuberculosis (15) as well as the rates of serious bacterial infections (16). An association between TNF- $\alpha$ antagonists and development of lymphoma has been repeatedly suggested but not unequivocally proven (17).

Other classes, such as non-steroidal anti-inflammatory drugs (NSAIDS, e.g., Celebrex and Vioxx) which act to inhibit the production of prostaglandins that promote inflammation, pain, and fever (9), have also been associated with serious side effects. A new appreciation for the resolution phase of inflammation, including the discovery and characterization of resolvins, protectins, and lipoxins, may presage a new class of safer anti-inflammatory agents that promote this pathway (10). However, metabolic instability and low oral bioavailability presently jeopardizes the pharmaceutical development of these compounds (11). Thus, the search for safe, pharmaceutically relevant anti-inflammatory agents to treat IMIDs continues to command considerable attention.

The abundant adrenal steroid DHEA (dehydroepiandrosterone) provides striking therapeutic benefits across a wide array of rodent models of IMID (12-14). Carefully controlled clinical trials of DHEA in patients with IMIDs have reported low efficacy and androgenic side effects (15-18), perhaps related to low oral bioavailability and differential interspecies metabolic rates and pathways $(13,19-21)$. Rodents rapidly metabolize exogenous DHEA into a surprisingly complex array of more highly oxidized metabolites (22), which may be responsible for the anti-inflammatory, immune regulating activity attributed to DHEA in rodents (14). Synthetic derivatives of active DHEA metabolites, with improved pharmaceutical properties and devoid of androgenic or estrogenic side effects, could potentially translate benefits observed in rodents into humans (14). The 7-hydroxyated metabolites of DHEA retain potent anti- 
inflammatory and immune modulating activity but are practically devoid of unwanted side effects (reviewed in ref. 23). Previously we showed that subcutaneous (s.c) injection of androstene- $3 \beta, 7 \beta, 17 \beta$-triol (AET) provided benefit in rodent models of ulcerative colitis (24) and experimental autoimmune encephalitis (25). By virtue of its anti-inflammatory activity, AET improved survival in a rodent model of traumatic shock (26). However, low oral bioavailability and metabolic instability of AET suggested limited pharmaceutical utility of this naturally occurring compound in humans. Therefore, analogues of AET were prepared and screened for improved pharmaceutical properties. The lead candidate, HE3286, was comprised of the core trihydroxylated androstene structure with the addition of an ethynyl group at the $17 \alpha$ position, which rendered the molecule orally bioavailable and stabilized the molecule to metabolism.

We previously reported that oral treatment with HE3286 inhibited collagen-induced arthritis in the mouse $(27,28)$. To extend and complement this finding we evaluated the effect of HE3286 in the Lewis rat model of adjuvant-induced arthritis (AIA) that represents another well known and validated preclinical model of human RA (29). We also replicated and confirmed the previously observed effects of HE3286 in the DBA mouse model of collagen antibodyinduced arthritis (CAIA) (28). In addition, we demonstrated that HE3286 is devoid of immunosuppressive properties both in in vitro and in vivo experimental settings using the mixed lymphocyte reaction response and the murine model of Coxsackie B3 virus-induced myocarditis, respectively.

\section{Materials and methods}

Drugs. Test compound $17 \alpha$-ethynyl-5-androstene-3ß, 7ß, 17ß-triol (HE3286) and vehicles (HERF202 or HERF405) were provided by HollisEden Pharmaceuticals (San Diego, CA). HERF202 contains 30\% B-cyclodextrin sulfobutyl ether sodium salt $(\mathrm{w} / \mathrm{v})$ in water. HERF405 contains $0.1 \%$ carboxymethylcellulose, $2 \%$ polysorbate 80 and $0.1 \%$ metabisulfite in phosphate-buffered saline $\mathrm{pH}$ 7.4. Control animals were treated with an equal volume of vehicle.

Animal care. Animals were purchased and housed in accordance with respective institutional guidelines and requirements of the relevant regulatory agencies. AIA rat and myocarditis mouse studies were performed by F.N. at UC. CAIA studies were performed by D.B. at UCSD as previously reported (28).

\section{Adjuvant-induced arthritis (AIA) in the rat}

Animals. A total of 40 male Lewis rats (Harlan Nossan, San Pietro al Natisone, Udine, Italy), ranging in weight from 162 to 215 grams, were used for the study. The rats were housed in a controlled environment (non-specific pathogen free) and provided with standard rodent chow and water.

Induction of arthritis and treatment. Adjuvant-induced arthritis (AIA) was induced in Lewis rats by a single s.c. injection $(0.1 \mathrm{ml})$ of heat-killed Mycobacterium tuberculosis H37Ra $(0.3 \mathrm{mg})$ in Freund's incomplete adjuvant into the base of the tail. Rats were divided randomly into two groups and treated orally (gavage) with either HE3286 (25 mg/kg) dissolved in vehicle (HERF202) or with vehicle alone, in a final volume of $0.125 \mathrm{ml}$. Two experimental treatments were considered, one with a therapeutic regime that occurs early in the disease process, and the second with a late therapeutic regime. For the early regimen, the rats were treated on day 8 post-immunization and in the late regimen, the rats were treated on day 15 postimmunization. In both cases treatments was given twice daily for 15 consecutive days. On the first day of treatment the rats were randomly allocated to the different experimental groups. Post-randomization analysis showed no significant differences in the mean arthritic score or for paw edema between the different groups.

Clinical assessment of AIA. Rats were evaluated daily for arthritis using a macroscopic scoring system: 0 , no signs of arthritis; 1, swelling and/or redness of the paw or one digit; 2 , two joints involved; 3 , more than two joints involved; and 4 , severe arthritis of the entire paw and digits. Arthritic index for each rat was calculated by adding the 4 scores of individual paws. Rats were weighed daily, beginning from the day of immunization.

Measurement of paw edema. Clinical disease severity was determined using plethysmography (model 7140; Ugo, Basel, Switzerland). The change in left hind paw volume was determined on a weekly basis.

Blood levels of IL-6 and TNF- $\alpha$. Circulating levels of IL-6 and TNF- $\alpha$ were measured by specific solid phase ELISA kits in undiluted plasma samples from the different groups of rats on day 0 (prior to immunization), 15th (at the beginning of the treatment) and at the end of the study. Inter and intrassay of variations were below 10 and 5\%, respectively. The assays were carried out according to the manufacturer's instructions (Bender MedSystems, Prodotti Gianni, Milan, Italy). The lower limit of sensitivity $31 \mathrm{pg} / \mathrm{ml}$ for IL-6 and $11.2 \mathrm{pg} / \mathrm{ml}$ for TNF- $\alpha$. To allow evaluation of statistical analysis, those samples with levels below the limit of sensitivity were assigned a value corresponding to the lower limit of sensitivity.

Determination of myeloperoxidase activity. Neutrophil infiltration into the inflamed joints was indirectly quantified using an MPO assay in both groups of rats treated with HE3286 or its vehicle at the end of the treatment (day 30). Briefly, the left hind-paw tissue was removed and snap frozen in liquid nitrogen. Upon thawing, the tissue $(0.1 \mathrm{~g}$ of tissue per $1.9 \mathrm{ml}$ buffer) was homogenized in buffer $(0.1 \mathrm{M} \mathrm{NaCl}$, $0.2 \mathrm{M} \mathrm{NaPO}_{4}$ and $\left.0.015 \mathrm{M} \mathrm{NaEDTA} ; \mathrm{pH} 4.7\right)$, centrifuged at $3000 \mathrm{x} \mathrm{g}$ for $10 \mathrm{~min}$ and the pellet subjected to hypotonic lysis $(1.5 \mathrm{ml}$ of $0.2 \% \mathrm{NaCl}$ solution followed $30 \mathrm{sec}$ later by addition of an equal volume of a solution containing $\mathrm{NaCl}$ $1.6 \%$ and glucose $5 \%$ ). After further centrifugation, the pellet was resuspended in $0.05 \mathrm{M} \mathrm{NaPO}_{4}$ buffer ( $\mathrm{pH}$ 5.4) containing $0.5 \%$ hexadecyltrimethylammonium bromide (HTAB) and rehomogenized. Aliquots $(1 \mathrm{ml})$ of the suspension were transferred into 1.5-ml Eppendorf tubes followed by three freeze-thaw cycles using liquid nitrogen. The aliquots were then centrifuged for $15 \mathrm{~min}$ at $3000 \mathrm{x} \mathrm{g}$, the pellet was 
resuspended to a volume of $1 \mathrm{ml}$ and samples of hind paw were assayed. Myeloperoxidase activity in the resuspended pellet was assayed by measuring the change in optical density at $450 \mathrm{~nm}$ using tetramethylbenzidine $(1.6 \mathrm{mM})$ and $\mathrm{H}_{2} \mathrm{O}_{2}$ $(0.5 \mathrm{mM})$.

\section{Collagen antibody induced arthritis (CAIA)}

Animals. DBA/1Lac/J male mice (5 per group) purchased from Jackson Laboratories (Bar Harbor, Maine) were treated (gavage) daily with either $0.1 \mathrm{ml}$ of vehicle (HERF202) or with HE3286 $(1,10$ or $40 \mathrm{mg} / \mathrm{kg})$, beginning on day 1 . Animals were treated for 14 days.

Induction and evaluation of CAIA. To induce arthritis, mice were administered $1 \mathrm{mg}$ of an anti-bCII antibody cocktail (Chondrex, Redmond, WA) intravenously on day -2 . On day 0 , animals were treated intraperitoneally (IP) with LPS $(12.5 \mu \mathrm{g})$, as in a previous study (28). The arthritic severity of the mice was evaluated as in our AIA studies.

Human mixed lymphocyte response (MLR) studies. Human blood samples were obtained from 3 healthy, fasting male volunteers, ranging from 23-31 years of age, who gave informed consent. The subjects had not used immune modulating, anti-allergic drugs or antibiotics in the three months prior to the study. Samples were drawn between 9 and 10 a.m. to avoid fluctuations in the circulating levels of hormones or cytokines.

Peripheral blood mononuclear cells (PBMC) were isolated by centrifugation on Ficoll-Hypaque (density 1.077, Biochrom AG, Berlin, Germany) gradients and resuspended in culture medium (RPMI-1640 supplemented with $2 \mathrm{mM}$ L-glutamine, penicillin $(100 \mathrm{U} / \mathrm{ml})$ and streptomycin $(100 \mu \mathrm{g} / \mathrm{ml})$ (Invitrogen s.r.l., Milan, Italy). Autologous (responder) inactivated plasma was used as vehicle at $10 \%$ concentration.

Five hundred thousand responder PBMC (PBMCr) and 500,000 allogeneic irradiated (30 Gy) stimulator PBMC (PBMCs) were mixed (ratio 1:1 in $200 \mu 1$ medium) and cultured for 6 days in a flat bottom 96-well plate (Nunc, Roskilde, Denmark). Test compound was dissolved in ethanol and then diluted to test concentrations $(0.3$ or $0.03 \mu \mathrm{M})$ with culture medium leading to a final solution containing $0.01 \%$ ethanol. Controls included vehicle, PBMCr and PBMCs cultured separately in the same vehicle at the same concentration. During the final $8 \mathrm{~h}$, the PBMC were pulsed with $1 \mu \mathrm{Ci} /$ well $\left[{ }^{3} \mathrm{H}\right]$-thymidine (Amersham, Milan, Italy). Cells were harvested and incorporation of radioactivity into DNA measured with a beta cell counter.

\section{Myocarditis induced by infection with Coxsackie B3 virus}

Animals. Four- to five-week-old BALB/c male mice were purchased from Harlan Nossan (Correzzana, Italy). They were kept under standard laboratory conditions (non-specific pathogen free) with ad libitum food and water and were allowed to adapt one week to their environment before study commencement.

Treatment. Mice were randomized by weight into 3 groups of 10 and treated by oral gavage with $80 \mathrm{mg} / \mathrm{kg} \mathrm{HE} 3286$ or vehicle HERF405, or IP with dexamethasone (Dex) at the dose of $0.4 \mathrm{mg} / \mathrm{kg}$ for 14 consecutive days. After 14 days of treatment the mice were infected intraperitoneally with $10^{5}$ plaque-forming units of purified CB3V. Animals were monitored twice daily, 7 days a week, for health status. Body weight was recorded daily. At day 28 post-infection, mice were sacrificed and the heart was weighed and submitted for histological analysis. The spleen was isolated and weighed.

Histology. At the end of the study, histopathological scores of hearts from groups of mice were evaluated as follows: $0=$ no lesions; 1 = foci of mononuclear cell inflammation associated with myocardial cell reactive changes without myocardial cell necrosis; 2 = inflammatory foci clearly associated with myocardial cell reactive changes; 3 = inflammatory foci clearly associated with myocardial cell necrosis and dystrophic calcification; 4 = extensive inflammatory infiltration, necrosis and dystrophic calcification.

\section{Statistical analysis}

Rat model of AIA. All data are expressed as the mean \pm SD. The significance of differences in arthritic scores was evaluated by Student's t-test vs. vehicle-treated rats. $\mathrm{p}<0.05$ was considered significant.

DBA mouse model of CAIA. The various pair-wise comparisons between each active group and placebo (at each time point) were tested for significant differences via the exact Van Elteren's test wherein scored data were stratified based on the median baseline score. Data imputation is implemented via LOCF.

Human MLR. The mean cpm of quadruplicate wells was calculated. The hypothesis of non-inferiority was not analyzed formally due to the small sample size (three subjects). Instead, an exploratory three-step procedure was executed as follows: First, an exact 95\% Hodges-Lehman confidence interval was constructed for the difference in population medians for each subject, regarding the replicates within that subject as independent. This step was then confirmed by means of the significance of non-inferiority of an active compound relative to a control via an exact one-sided MannWhitney test, per subject as above (30). Second, a decision was made as to whether each subject meets the non-inferiority criteria. Finally, the percentage of subjects adjudicated as cases of non-inferiority was calculated, with its attendant exact Blyth-Still-Casella confidence interval for the total fraction of subjects that, under similar experimental conditions, would show non-inferiority. This is a measure of the strength of the evidence at the population level inferred from the three cases (31).

Myocarditis. The various pairwise comparisons for body weight, spleen weight, and heart weight were tested for significant differences via the Wilcoxon-Mann-Whitney test. Histology scores were tested via the Freeman-Halton test.

\section{Results}

Effect of HE3286 in the rat model of AIA

Study I. Effect of early therapeutic treatment with HE3286 on 

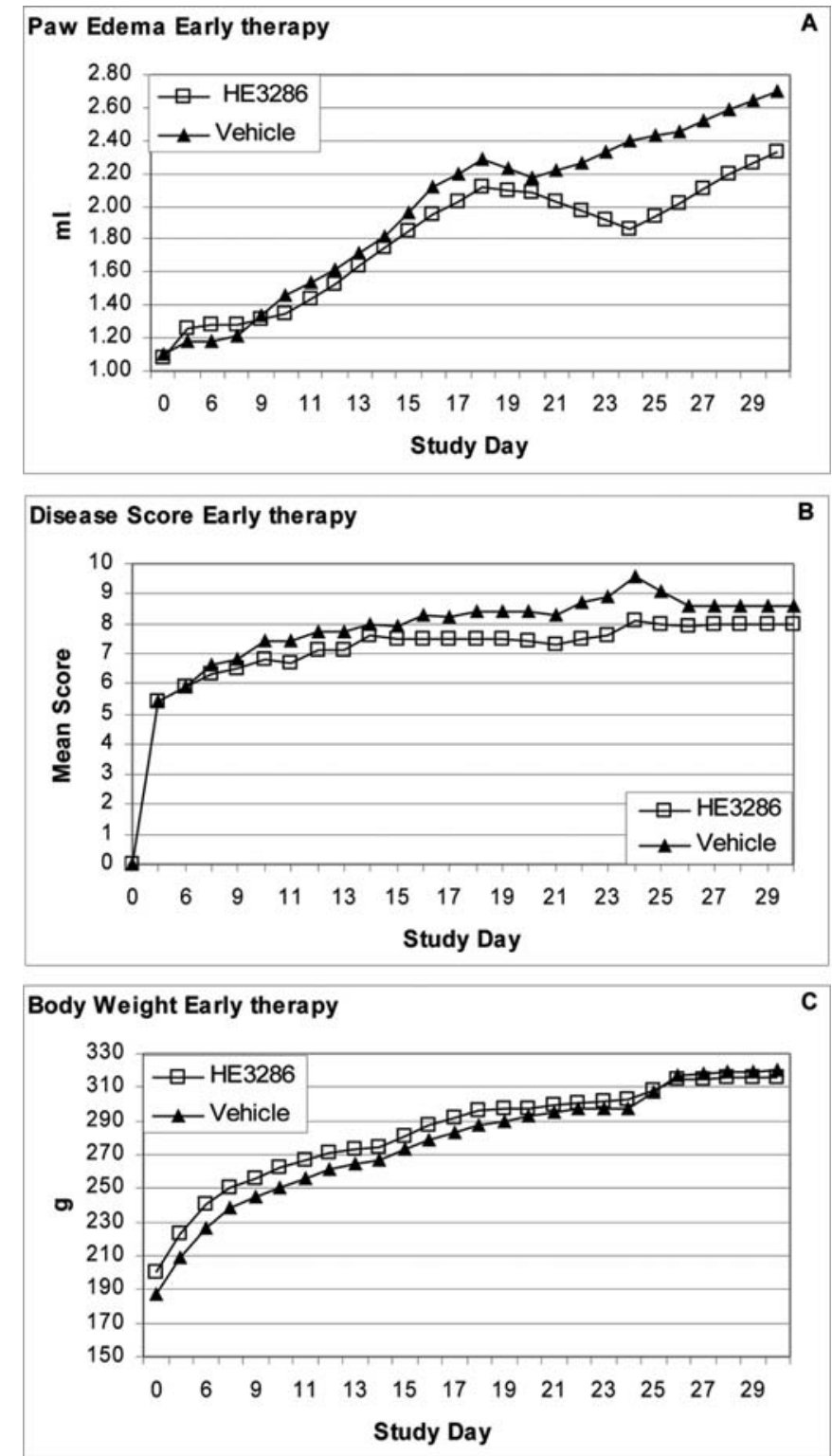

Figure 1. Effect of early therapeutic treatment with HE3286 in the rat model of AIA. Arthritis was induced in male Lewis rats (10 per group) by a single (s.c.) injection $(0.1 \mathrm{ml})$ of heat-killed Mycobacterium tuberculosis $\mathrm{H} 37 \mathrm{Ra}$ $(0.3 \mathrm{mg})$ in Freund's incomplete adjuvant into the base of the tail. On day 8 (post-immunization to induce AIA), when the rats showed clinical signs of arthritis, twice-daily treatments (gavage) began with either HE3286 (25 mg/ $\mathrm{kg}$ ) or vehicle alone $(125 \mu \mathrm{l})$ and were continued for 15 consecutive days. Paw edema and disease score were assessed daily as described in Materials and methods. Data are expressed as median paw edema (A) and median disease score per animal (B) \pm standard deviation. (C) The body weight variation during the study period.

the development of AIA. The data in Fig. $1 \mathrm{~A}$ and $\mathrm{B}$ demonstrated a time-dependent increase in both left hind paw volume $(\mathrm{ml})$ and clinical arthritic score that were induced by adjuvant in vehicle-treated rats The arthritis incidence in these animals was 100\% (10/10). The rats treated with HE3286 exhibited significantly lower paw edema than vehicle-treated rats from day 22 to the end of the study on day 30 (Fig. 1A). HE3286 also significantly reduced the arthritic score of the treated rats as compared to vehicle from days 18-25 (Fig. 1B). The fluctuations of body weight seen in
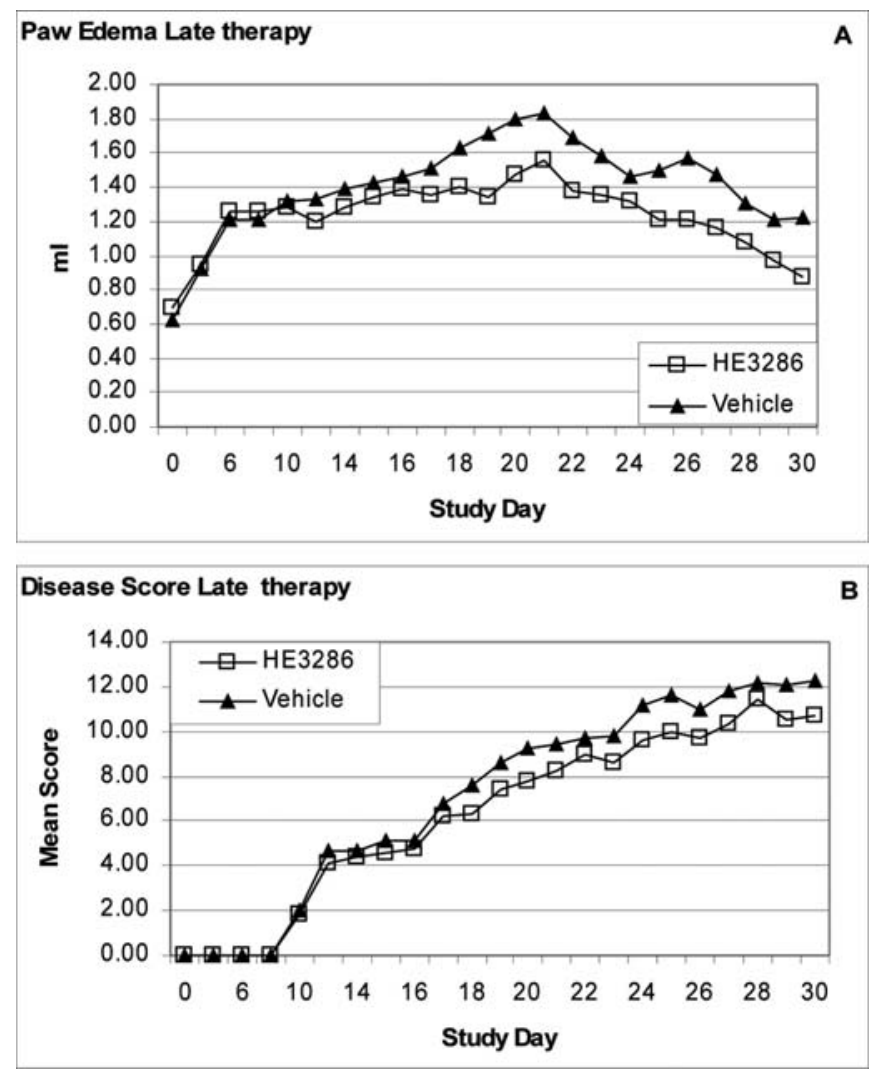

Figure 2. Effect of late therapeutic treatment with HE3286 in the rat model of AIA. Arthritis was induced in male Lewis rats (10 per group) by a single (s.c.) injection $(0.1 \mathrm{ml})$ of heat-killed Mycobacterium tuberculosis H37Ra $(0.3 \mathrm{mg})$ in Freund's incomplete adjuvant into the base of the tail. On day 15 (post-immunization to induce AIA), when the rats showed clinical signs of well-established arthritis, twice-daily treatments (gavage) began with either HE3286 $(25 \mathrm{mg} / \mathrm{kg})$ or vehicle alone $(125 \mu 1)$ and were continued for 15 consecutive days. Paw edema and disease score were assessed daily as described in Materials and methods. Data are expressed as median paw edema (A) and median disease score per animal (B) \pm SD.

HE3286-treated control rats were comparable to those observed in the vehicle-treated controls (Fig. 1C).

Study II. Effect of late therapeutic treatment with HE3286 on the development of AIA. The efficacy of early therapeutic treatment on development of rat AIA prompted us to evaluate the efficacy of this compound when first applied under a fully therapeutic regime to rats with advanced clinical signs of arthritis. To this aim the rats were first treated with HE3286 from day 15 post-immunization. Also upon this late therapeutic treatment HE3286 exhibited powerful anti-arthritogenic effects and it was capable of favourably influencing the course of rat AIA. In fact, relative to vehicle-treated controls, the rats treated with HE3286 exhibited significantly lower paw edema from day 19 until the end of the study on day 30 (Fig. 2A) with exception of non-significant reductions vs. on days 23 and 24. HE3286 also significantly reduced the arthritic score of the treated rats as compared to vehicle from days 19-30 (Fig. 2B) with exception on day 22 where the reduction of the arthritic score was close to, but did not reach statistical significance. The fluctuations in body weight seen in HE3286treated control rats were comparable to those observed in the vehicle-treated (data not shown). 

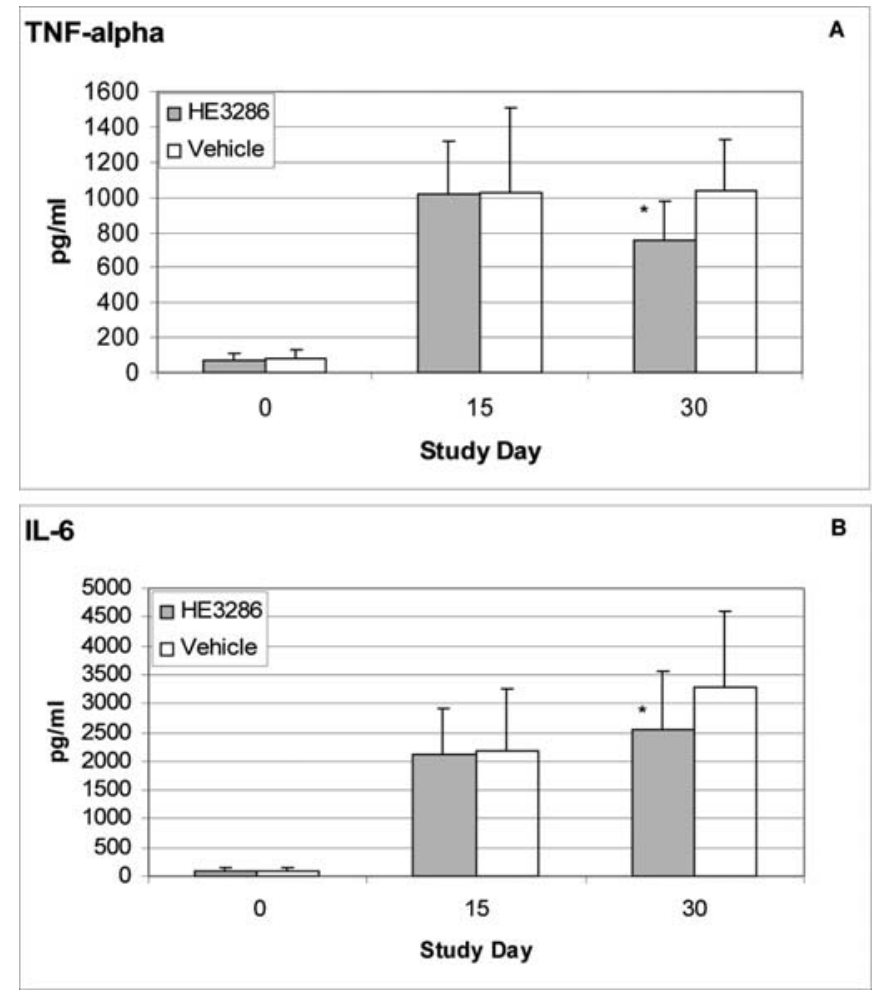

Figure 3. Effects of late therapeutic treatment with HE3286 on reduction of IL-6 and TNF- $\alpha$ blood levels in the AIA rat model. Cytokine blood levels in adjuvant-induced arthritis (AIA) on day 30 after adjuvant injection after 15 days of treatment with HE3286. (A) Tumor necrosis factor- $\alpha$ (TNF- $\alpha$ ) levels in sera $(n=10)$ and (B) IL-6 levels in sera $(n=10)$. Values were measured by ELISA and are expressed as the mean \pm SD. ${ }^{*} \mathrm{p}<0.05$.

Late therapeutic treatment with HE3286 reduced blood levels of IL-6 and TNF- $\alpha$ that were found to be elevated in vehicle-treated controls.

IL-6 and TNF- $\alpha$ were undetectable in most of these rats before immunization and increased thereafter with progression of the disease. A fluctuation similar to that seen in the vehicletreated rats was also seen in the group of rats assigned to HE3286 treatment. However, when re-measured at the end of the study after 15 consecutive days of treatment with HE3286 the circulating levels of these cytokines were significantly lower in the treated rats as compared to those receiving the vehicle (Fig. 3A and B).

MPO activity was markedly increased, on day 30 in AIAimmunized rats treated with the vehicle. Late therapeutic treatment with HE3286 reduced the MPO activity in the hind-paw homogenate. The treatment with HE3286 for 15 consecutive days significantly reduced the MPO activity compared to vehicle-treated rats (Fig. 4).

Effect of HE3286 in the DBA mouse model of CAIA. In the CAIA model, daily treatment with HE3286 (40 mg/kg) produced benefit when compared to animals receiving either vehicle or 1 or $10 \mathrm{mg} / \mathrm{kg} \mathrm{HE} 3286$ (Fig. 5). In this study, CAIA began to develop by day 3 (score $<4$ ) in both vehicle- and HE3286-treated. Disease peaked in all groups on days 7-8, where average scores were between 9 and 12 in both the

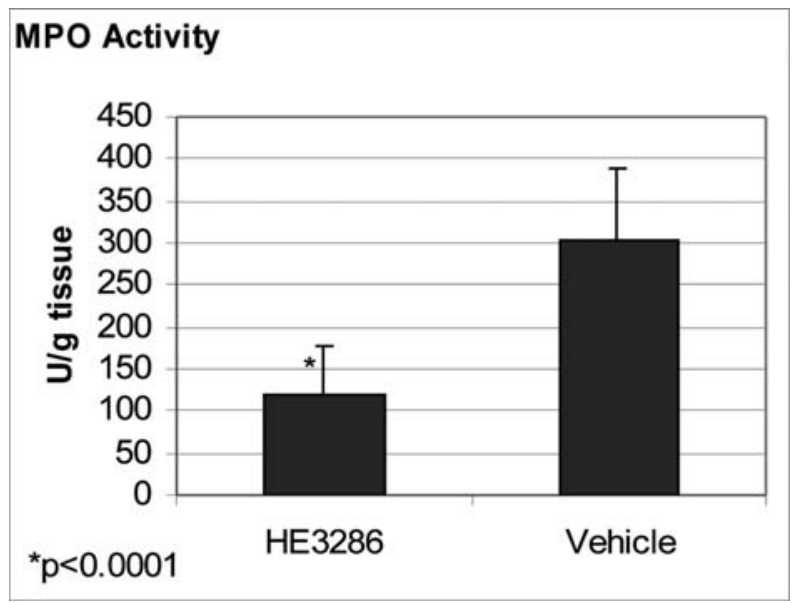

Figure 4. Effects of late therapeutic treatment with HE3286 on reduction of MPO activity in the AIA rat model. MPO activity in the left hind-paw tissue on day 30 after adjuvant injection after 15 days of treatment with HE3286. $\mathrm{n}=10$ per group. Optical density values were measured using tetramethylbenzidine. Data are expressed as the mean \pm SD.

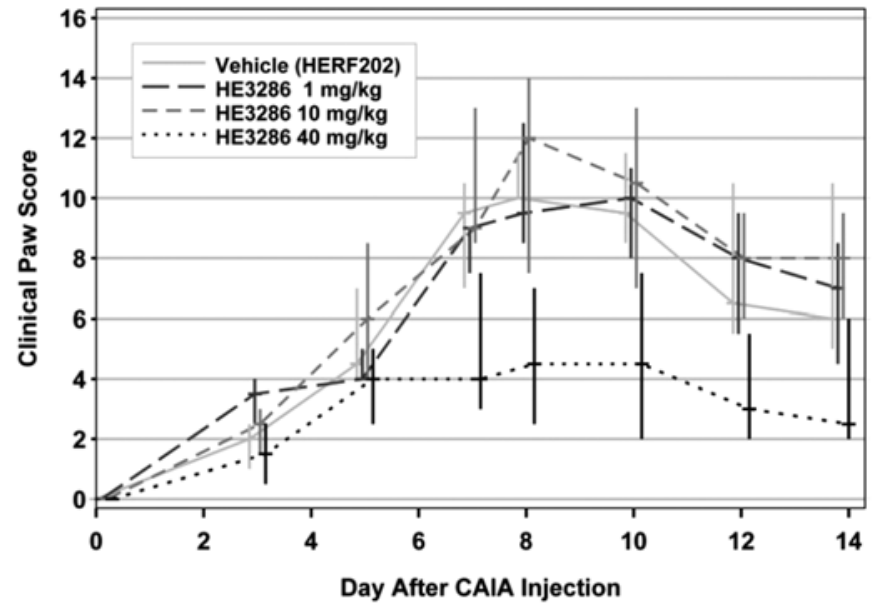

Figure 5. Effect of HE3286 in the DBA mouse model of CAIA. DBA male mice (5 per group) were administered $1 \mathrm{mg}$ of an anti-bCII antibody cocktail intravenously on day -2 . On day 0 , animals were treated (IP) with LPS $(12.5 \mu \mathrm{g})$. Animals were treated daily with either $0.1 \mathrm{ml}$ of vehicle or with HE3286 $(1,10$ or $40 \mathrm{mg} / \mathrm{kg})$ beginning on day 1 . Data are expressed as the group's average clinical paw score \pm SD.

vehicle-treated and lower dose (1 and $10 \mathrm{mg} / \mathrm{kg}$ ) HE3286treated groups. In contrast, the high dose $(40 \mathrm{mg} / \mathrm{kg})$ became statistically lower on day $4(\mathrm{p}<0.031)$ and remained lower for the remainder of the study (day 14). Dose response trends were clearly detectable and statistically significant starting at day 4 . This study therefore fully confirms a similar study with HE3286 in this model that has been recently reported (33).

Effect of HE3286 in human MLR. Minimal proliferative responses (>2000 CPM) were observed at the end of the culture period in individual cultures of control responder and stimulator PBMCs (obtained from each of three donors R1, $\mathrm{R} 2$ and R3). In contrast, a marked proliferative response was observed when PBMCr and PBMCs were cultured together with or without vehicle (Fig. 6). Overall, there was no 
Table I. Effects of HE3286 on body weight variations, spleen and heart weights and heart histological score in male BALB/C mice infected with Coxsackie B3 virus.

\begin{tabular}{|c|c|c|c|c|c|c|}
\hline & \multicolumn{3}{|c|}{ Variation body weight (\%) } & \multirow{2}{*}{$\begin{array}{l}\text { Spleen weight } \\
(\mathrm{mg})\end{array}$} & \multirow{2}{*}{$\begin{array}{l}\text { Heart weight } \\
(\mathrm{mg}) \\
\text { Day } 42\end{array}$} & \multirow{2}{*}{$\frac{\text { Histo score }}{\text { Day } 42}$} \\
\hline & $\begin{array}{c}\text { Day 0 } \\
\text { Start of } \\
\text { treatment }\end{array}$ & $\begin{array}{l}\text { Day } 14 \\
\text { End of treatment } \\
\text { before infection }\end{array}$ & $\begin{array}{c}\text { Day } 42 \\
\text { sacrifice }\end{array}$ & & & \\
\hline HE3286 & 0 & $-1.0 \pm 8.3$ & $27.1 \pm 8.9$ & $99.6 \pm 20.9$ & $139.4 \pm 12.6$ & $0.0 \pm 0.0$ \\
\hline Dex & 0 & $-3.6 \pm 3.4$ & $24.3 \pm 5.5$ & $96.8 \pm 9.8$ & $143.7 \pm 13.4$ & $0.1 \pm 0.3$ \\
\hline Vehicle & 0 & $13.1 \pm 4.3$ & $28.7 \pm 7.6$ & $98.6 \pm 10.4$ & $152.9 \pm 18.1$ & $0.6 \pm 0.8$ \\
\hline SHAM & 0 & $18.2 \pm 12.1$ & $37.7 \pm 10.5$ & $82.1 \pm 3.8$ & $147.1 \pm 9.9$ & $0.0 \pm 0.0$ \\
\hline
\end{tabular}

BALB/c male mice ( $\mathrm{n}=10$ per group) were treated orally (gavage) with HE3286 (80 mg/kg) or with vehicle alone for 14 consecutive days; Dex was administered IP under the same experimental conditions. Body weight was measured daily and, after 14 days of treatment, the mice were infected intraperitoneally with $10^{5}$ plaque-forming units of purified virus. Animals were monitored twice daily, 7 days a week for health status. At day 28 post-infection, the mice were sacrificed, the spleen and heart were weighed and the heart histologically analyzed.

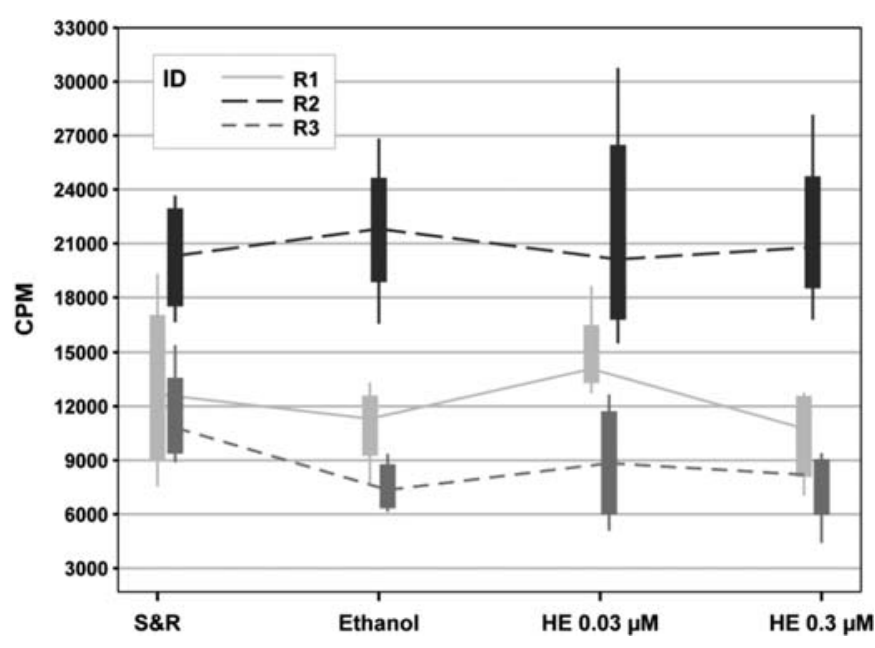

Figure 6. Effect of HE3286 in human MLR. Five hundred thousand responders PBMC (PBMCr) and 500,000 allogeneic irradiated (30 Gy) stimulator PBMC (PBMCs) from three different volunteers (R1, R2 and R3) were mixed (at a ratio of 1:1 in $200 \mu 1$ medium) and cultured for 6 days in a flat bottom, 96-well plates $( \pm 0.3 \mu \mathrm{M}$ or $0.03 \mu \mathrm{M}$ HE3286). HE3286 was dissolved in ethanol and then diluted to desired concentration with culture medium leading to a final solution containing $0.01 \%$ ethanol. Controls included $0.01 \%$ ethanol alone, and PBMCr and PBMCs cultured separately. During the last $8 \mathrm{~h}$ of the culture period the PBMC were pulsed with $1 \mu \mathrm{Ci} /$ well $\left[{ }^{3} \mathrm{H}\right]$ thymidine. Cells were harvested and radioactivity incorporation measured with a beta cell counter. Data are expressed as CPM. CPMs, counts per minute; ID, subject identification code; S\&R, stimulator and responder jointly; HE, HE3286. The bottom and top edges of the box are located at the sample 25 th and 75 th percentiles. The vertical lines, or whiskers, are drawn from the box to the most extreme point within 1.5 interquartile ranges. The line connecting the treatments passes through the respective median CPM for each treatment.

profound suppressive influence of any HE3286 concentration tested.

Effect of HE3286 in myocarditis induced by CB3V. During the 14-day treatment period, Dex- and HE3286-treated mice showed relatively no weight gain while sham-treated mice and vehicle-treated mice averaged 13 and $18 \%$ gain in body weight, respectively (Table I). This difference may have been due to a genuine effect of HE3286. If so, this effect of HE3286 appears to be strain-specific, since it was not observed in other strains [e.g., DBA mice, SJL mice or NOD mice (unpublished data)]. However, beginning two days following CB3V infection through the end of the study, the increased change in body weight from day 14 significantly $(\mathrm{p}<0.05)$ favoured both the Dex- and HE3286-treated mice when compared to the vehicle-treated mice.

As expected, the infection with CVB3 induced a significant $(\mathrm{p}<0.05)$ increase in spleen weight in vehicle-treated mice as compared to the healthy (sham) mice. However, two weeks of daily pre-treatments with either HE3286 or Dex prior to viral challenge did not appear to exert any profound effects on the subsequent course of the infection as no significant differences were observed in spleen or heart weights compared to the mice pre-treated with the vehicle alone. However, there was a trend $(\mathrm{p}=0.093)$ towards reduced heart weights (suggestive of reduced inflammation) in the HE3286-treated group compared to the vehicle-treated group (139.4 mg \pm 12.6 vs. $152.9 \mathrm{mg} \pm 18.1$ ). This difference could not be attributed to any significant difference in body weights between these groups at the end of the study.

The histological examination of the heart showed only low numbers of cellular infiltration foci and some calcified areas in the group of mice that had been pre-treated with vehicle (Table I). However, histology did show a trend ( $p=0.087)$ toward lower scores in hearts of animals that had been pre-treated with HE3286 compared to those treated with vehicle alone, also suggesting a slight benefit from pre-treatment.

\section{Discussion}

The data presented herein show for the first time that HE3286, a synthetic derivative of AET, provided clinical benefit in rodent models of AIA with a simultaneous reduction of MPO activity and diminished blood levels of IL- 6 and TNF- $\alpha$. We also confirm the finding of a previous study (28) on the 
efficacy of this compound to ameliorate the course of CAIA. In addition, we show herein that HE3286 was not immune suppressive in MLR studies and that extended pre-treatments were safe and may even have provided benefit in the murine model of myocarditis induced by CB3V infection. The present studies confirm the anti-inflammatory activity reported for HE3286 in our previous publications $(23,24,26)$.

Our observation that HE3286 provides benefit in the rat model of AIA, even when treatment is delayed until disease is well established, is in agreement with our previous studies in the type II CIA DBA mouse model of arthritis (28). This observation has strong significance for the potential translation of these findings to the clinical setting as it has been shown that a compound's therapeutic efficacy, rather than prophylactic efficacy, in AIA and CIA models is more predictive of clinical efficacy in human RA than data from either model alone (29).

In contrast to CIA, no single antigen has been identified that is deemed responsible for the arthritogenic response in the AIA model (32). Rather, the induction of adjuvant arthritis has been attributed to a mycobacterial cell wall component, muramyl-dipeptide, which is immunostimulatory but does not evoke a specific immune response (33). In the rat model of AIA, the chief pathological features of adjuvant arthritis include edema, mononuclear and polymorphonuclear cell joint infiltration, pannus formation, periostitis and erosion of cartilage and bone. Hence the reduced MPO activity seen in HE3286-treated rats as compared to vehicle-treated controls is probably secondary to reduced neutrophil influx into the inflamed paws. This is consistent with the clinical observation of both a reduced arthritic score as well as paw edema observed upon treatment with HE3286.

Rat AIA exhibits immunopathogenic and pharmacological similarities with human RA including the increased blood levels of IL- 6 and TNF- $\alpha$ during the course of the disease $(34,35)$ and the favourable response to immunosuppressive and anti-inflammatory drugs used in RA such as cyclosporin A (36), leflunomide methotrexate and indomethacin $(37,38)$. As in human RA (39), cyclooxygenase (COX) II appears to be involved in the pathogenesis of rat AIA since occurrence of arthritis is associated with local production of PGE2 and up-regulated expression of COX-2, IL- 6 and TNF- $\alpha$ mRNAs in the affected paws (38). Selective inhibition of COX-2 reverses clinical occurrence of AIA and expression of COX-2 and IL-6. This resembles our present observation of concomitant inhibition of clinical signs of AIA and reduced blood levels of IL-6 (and TNF- $\alpha$ ) in the rats treated with HE3286. These findings are also consistent with our studies in murine type II CIA where HE3286-mediated clinical benefit was associated with decreased production of pro-inflammatory cytokines such as TNF- $\alpha$, IL-6, and IL-17 (28). The above findings may indicate that the anti-arthritogenic capacities of HE3286 may be at least partly mediated by its capacity to down-regulate the synthesis of proinflammatory cytokines involved in arthritogenic pathways such as IL-6 and TNF- $\alpha$.

In agreement with our earlier studies (28), we report herein the significant benefit of HE3286 treatment in the CAIA model, where the typical disease course involves a short period of acute disease followed by gradual resolution in joint inflammation (40). The CAIA model permits an analysis of the contribution of non- $\mathrm{T}$ and $\mathrm{B}$ cell compartments to the pathogenesis of experimental RA. The observation that HE3286 provides benefit in these 3 models of arthritis such as CIA, AIA, and CAIA that recognize differences in pathogenetic pathways probably relates to a multifaceted mechanism of action and suggests that there is good potential for success in the treatment of human RA.

Our previous studies with AET showed benefit in rodent models of ulcerative colitis and multiple sclerosis $(23,24)$. Thus, a broad based anti-inflammatory activity is perhaps central to the activities of HE3286 in auto-immune disease. However, the exact mechanism by which HE3286 mediates these effects remains elusive. Potential mechanisms of action for 7-hydroxy steroids have been grouped into four broad categories, including gating (ligand inactivation), modulation of ion channels, interaction with atypical receptors, and modulation of steroidogenic enzymes (41). Non-genomic cell surface receptors are also a possible target, as may be the case with DHEA $(42,43)$ and estradiol (44). Potential targets within each of these categories are currently under consideration. In terms of potential cellular targets, our previous studies (28) associated the activity of HE3286 with increased regulatory $\mathrm{T}$ cells, thought to play pivotal roles in the maintenance of immune homeostasis and in the prevention of autoimmune disease (45). We also found reductions in key pro-inflammatory cytokines including IL-17 and IL-6. The role of these cytokines in the biology of regulatory $\mathrm{T}$ cells $(46,47)$, the transition from acute to chronic inflammation (48), and in IMID (45) is well established.

HE3286 was not found to be immune suppressive in vivo or in vitro in the present studies. Also while no immune suppressive effect of Dex could be demonstrated at the end of our CVB3 study, HE3286 was not found to be immune suppressive in any of the classic models of immune suppression, such as mitogen-induced lymphocyte proliferation, delayed type hypersensitivity responses or the popliteal lymph node assay, as reported previously (28). HE3286 does not appear to interact (either via binding or transactivation) with any of the known nuclear hormone receptors, including the glucocorticoid or sex steroid receptors (Riveros-Flores et al, unpublished data).

Whatever the exact mechanism of action, HE3286 has demonstrated attractive pharmaceutical properties including surprisingly good oral bio-availability (25\%) in rodents, primates and humans and resistance to metabolism (HollisEden Pharmaceuticals, unpublished data). Recent observations in ongoing phase I/II trials suggest an attractive half-life in humans $(8 \mathrm{~h})$ compared to rodents, and demonstrate that putative efficacious blood levels are attainable with doses in the same range as commonly prescribed glucocorticoids. The anti-inflammatory activity of HE3286 resembles glucocorticoids, but has a distinctly different safety profile consistent with use in disease settings of chronic inflammation, where administration over extended periods of time is desirable. In this regard, HE3286 appears to be bone-sparing in both in vitro and in vivo models (Malik et al, unpublished data). Taken together with the anti-inflammatory activity of HE3286 observed in several animal models, the data presented herein strengthen the application of this compound to RA patients. In addition, the apparent lack of immunosuppressive effects of HE3286 may represent an important advantage for the 
prolonged use of this compound in other both systemic and organ-specific autoimmune diseases including systemic lupus erythematosus, multiple sclerosis, Guillain Barrè syndrome, inflammatory bowel disease and type 1 diabetes. HE3286 is currently in phase I/II clinical trials in RA and ulcerative colitis, and may represent a novel, first in class antiinflammatory agent, with similar applications as the corticosteroids and cyclooxygenase inhibitors, but with an improved safety profile.

\section{References}

1. Kuek A, Hazleman BL and Ostor AJ: Immune-mediated inflammatory diseases (IMIDs) and biologic therapy: a medical revolution. Postgrad Med J 83: 251-260, 2007.

2. Wislowska $M$ and Jakubicz D: Preliminary evaluation in rheumatoid arthritis activity in patients treated with TNF-alpha blocker plus methotrexate versus methotrexate or leflunomide alone. Rheumatol Int 27: 641-647, 2007.

3. Sandborn WJ and Hanauer SB: Antitumor necrosis factor therapy for inflammatory bowel disease: a review of agents, pharmacology, clinical results, and safety. Inflamm Bowel Dis 5: 119-133, 1999.

4. Monk JP, Phillips G, Waite R, Kuhn J, Schaaf LJ, Otterson GA, Guttridge D, Rhoades C, Shah M, Criswell T, Caligiuri MA and Villalona-Calero MA: Assessment of tumor necrosis factor alpha blockade as an intervention to improve tolerability of doseintensive chemotherapy in cancer patients. J Clin Oncol 24: 1852-1859, 2006.

5. Kong JS, Teuber SS and Gershwin ME: Potential adverse events with biologic response modifiers. Autoimmun Rev 5: 471-485, 2006.

6. Schacke H, Docke WD and Asadullah K: Mechanisms involved in the side effects of glucocorticoids. Pharmacol Ther 96: 23-43, 2002.

7. Callen JP: Complications and adverse reactions in the use of newer biologic agents. Semin Cutan Med Surg 26: 6-14, 2007.

8. Berger JR, Houff S: Opportunistic infections and other risks with newer multiple sclerosis therapies. Ann Neurol 65: $367-$ $377,2009$.

9. Turini ME and DuBois RN: Cyclooxygenase-2: a therapeutic target. Annu Rev Med 53:35-57, 2002.

10. Serhan CN, Chiang N and Van Dyke TE: Resolving inflammation: dual anti-inflammatory and pro-resolution lipid mediators. Nat Rev Immunol 8: 349-361, 2008.

11. Serhan CN: Resolution phase of inflammation: novel endogenous anti-inflammatory and proresolving lipid mediators and pathways. Annu Rev Immunol 25: 101-137, 2007.

12. Webb SJ, Geoghegan TE, Prough RA and Michael Miller KK: The biological actions of dehydroepiandrosterone involves multiple receptors. Drug Metab Rev 38: 89-116, 2006.

13. Svec F and Porter JR: The actions of exogenous dehydroepiandrosterone in experimental animals and humans. Proc Soc Exp Biol Med 218: 174-191, 1998.

14. Auci DL, Ahlem C, Li M, Trauger R, Dowding C, Paillard F, Frincke $\mathrm{J}$ and Reading CL: The immunobiology and therapeutic potential of androstene hormones and their synthetic derivatives: novel anti-inflammatory and immune regulating steroid hormones. Mod Asp Immunobiol 3: 64-70, 2003.

15. Huppert FA, Van Niekerk JK and Herbert J: Dehydroepiandrosterone (DHEA) supplementation for cognition and wellbeing. Cochrane Database Syst Rev 2: CD000304, 2000.

16. Sirrs SM and Bebb RA: DHEA: panacea or snake oil? Can Fam Physician 45: 1723-1728, 1999

17. Celec P and Starka L: Dehydroepiandrosterone - is the fountain of youth drying out? Physiol Res 52: 397-407, 2003.

18. Petri MA, Mease PJ, Merrill JT, Lahita RG, Iannini MJ, Yocum DE, Ginzler EM, Katz RS, Gluck OS, Genovese MC, Van Vollenhoven R, Kalunian KC, Manzi S, Greenwald MW, Buyon JP, Olsen NJ, Schiff MH, Kavanaugh AF, Caldwell JR, Ramsey-Goldman R, St Clair EW, Goldman AL, Egan RM, Polisson RP, Moder KG, Rothfield NF, Spencer RT, Hobbs K, Fessler BJ, Calabrese LH, Moreland LW, Cohen SB, Quarles BJ, Strand V, Gurwith M and Schwartz KE: Effects of prasterone on disease activity and symptoms in women with active systemic lupus erythematosus. Arthritis Rheum 50: 2858-2868, 2004.
19. Leblanc M, Labrie C, Belanger A, Candas B and Labrie F: Bioavailability and pharmacokinetics of dehydroepiandrosterone in the cynomolgus monkey. J Clin Endocrinol Metab 88: 4293-4302, 2003.

20. Buster JE, Casson PR, Straughn AB, Dale D, Umstot ES, Chiamori N and Abraham GE: Postmenopausal steroid replacement with micronized dehydroepiandrosterone: preliminary oral bioavailability and dose proportionality studies. Am J Obstet Gynecol 166: 1163-1170, 1992.

21. Fitzpatrick JL, Ripp SL, Smith NB, Pierce WM Jr and Prough RA: Metabolism of DHEA by cytochromes P450 in rat and human liver microsomal fractions. Arch Biochem Biophys 389: 278-287, 2001.

22. Marwah A, Marwah P and Lardy H: Ergosteroids. VI. Metabolism of dehydroepiandrosterone by rat liver in vitro: a liquid chromatographic-mass spectrometric study. J Chromatogr B Biomed Sci Appl 767: 285-299, 2002.

23. Auci DL, Reading CL and Frincke JM: 7-Hydroxy androstene steroids and a novel synthetic analogue with reduced side effects as a potential agent to treat autoimmune diseases. Autoimmun Rev 8: 369-372, 2009.

24. Ahlem C, Auci D, Mangano K, Reading C, Frincke J, Stickney D and Nicoletti F: HE3286: a novel sythetic steroid as an oral treatment for autoimmune disease. Ann NY Acad Sci 1173: 781-790, 2009.

25. Offner H, Zamora A, Drought H, Matejuk A, Auci D, Morgan E, Vandenbark A and Reading C: A synthetic androstene derivative and a natural androstene metabolite inhibit relapsing-remitting EAE. J Neuroimmunol 130: 128-139, 2002.

26. Marcu AC, Kielar ND, Paccione KE, Barbee RW, Carter H, Ivatury RR, Diegelmann RF, Ward KR and Loria RM: Androstenetriol improves survival in a rodent model of traumatic shock. Resuscitation 71: 379-386, 2006.

27. Auci D, Kaler L, Subramanian S, Huang Y, Frincke J, Reading C and Offner H: A new orally bioavailable syntheic androstene inhibits collagen-induced arthritis in the mouse. Ann NY Acad Sci 1110: 630-640, 2007.

28. Offner H, Firestein GS, Boyle DL, Pieters R, Frincke JM, Garsd A, White SK, Reading CL and Auci DL: An orally bioavailable synthetic analog of an active dehydroepiandrosterone metabolite reduces established disease in rodent models of rheumatoid arthritis. J Pharmacol Exp Ther 329: 1100-1109, 2009.

29. Hegen M, Keith JC Jr, Collins M and Nickerson-Nutter CL: Utility of animal models for identification of potential therapeutics for rheumatoid arthritis. Ann Rheum Dis 67: 1505-1515, 2008.

30. Conover WJ: Practical Nonparametric Statistics. 3rd edition, John Wiley \& Sons Inc., New York, NY, pp271-364, 1999.

31. Casella G: Refining binomial confidence intervals. Can J Stat 14: 113-129, 1986

32. Holmdahl R, Goldschmidt TJ, Kleinau S, Kvick C and Jonsson R: Arthritis induced in rats with adjuvant oil is a genetically restricted, alpha beta T-cell dependent autoimmune disease. Immunology 76: 197-202, 1992.

33. Kohashi O, Aihara K, Ozawa A, Kotani S and Azuma I: New model of a synthetic adjuvant, N-acetylmuramyl-L-alanyl-Disoglutamine-induced arthritis: clinical and histologic studies in athymic nude and euthymic rats. Lab Invest 47: 27-36, 1982.

34. Szekanecz Z, Halloran MM, Volin MV, Woods JM, Strieter RM, Kenneth Haines G III, Kunkel SL, Burdick MD and Koch AE: Temporal expression of inflammatory cytokines and chemokines in rat adjuvant-induced arthritis. Arthritis Rheum 43: 1266-1277, 2000 .

35. Badolato R and Oppenheim JJ: Role of cytokines, acute-phase proteins, and chemokines in the progression of rheumatoid arthritis. Semin Arthritis Rheum 26: 526-538, 1996.

36. Haynes DR, Gadd SJ, Whitehouse MW, Mayrhofer G and Vernon-Roberts B: Complete prevention of the clinical expression of adjuvant-induced arthritis in rats by cyclosporin-A and lobenzarit: the regulation of lymph node cell populations and cytokine production. Inflamm Res 45: 159-165, 1996.

37. Magari K, Miyata S, Nishigaki F, Ohkubo Y and Mutoh S: Comparison of anti-arthritic properties of leflunomide with methotrexate and FK506: effect on T cell activation-induced inflammatory cytokine production in vitro and rat adjuvantinduced arthritis. Inflamm Res 53: 544-550, 2004.

38. Anderson GD, Hauser SD, McGarity KL, Bremer ME, Isakson PC and Gregory SA: Selective inhibition of cyclooxygenase (COX)-2 reverses inflammation and expression of COX-2 and interleukin 6 in rat adjuvant arthritis. J Clin Invest 97: 2672-2679, 1996. 
39. Matsumoto A, Melian A, Shah A and Curtis SP: Etoricoxib versus naproxen in patients with rheumatoid arthritis: a prospective, randomized, comparator-controlled 121-week trial. Curr Med Res Opin 23: 2259-2268, 2007.

40. Nandakumar KS, Svensson L and Holmdahl R: Collagen type II-specific monoclonal antibody-induced arthritis in mice: description of the disease and the influence of age, sex, and genes. Am J Pathol 163: 1827-1837, 2003.

41. Lathe R: Steroid and sterol 7-hydroxylation: ancient pathways. Steroids 67: 967-977, 2002.

42. Liu D and Dillon JS: Dehydroepiandrosterone activates endothelial cell nitric oxide synthase by a specific plasma membrane receptor coupled to Galpha i2,3. J Biol Chem 277: 21379-21388, 2002

43. Waterhouse RN, Chang RC, Atuehene $\mathrm{N}$ and Collier TL: In vitro and in vivo binding of neuroactive steroids to the sigma-1 receptor as measured with the positron emission tomography radioligand [18F]FPS. Synapse 61: 540-546, 2007.
44. Prossnitz ER, Sklar LA, Oprea TI and Arterburn JB: GPR30: a novel therapeutic target in estrogen-related disease. Trends Pharmacol Sci 29: 116-123, 2008.

45. Zwar TD, van Driel IR and Gleeson PA: Guarding the immune system: Suppression of autoimmunity by CD4CD25 immunoregulatory T cells. Immunol Cell Biol 84: 487-501, 2006.

46. Lohr J, Knoechel B, Wang JJ, Villarino AV and Abbas AK: Role of IL-17 and regulatory T lymphocytes in a systemic autoimmune disease. J Exp Med 203: 2785-2791, 2006.

47. Wan S, Xia C and Morel L: IL-6 produced by dendritic cells from lupus-prone mice inhibits $\mathrm{CD} 4{ }^{+} \mathrm{CD} 25^{+} \mathrm{T}$ cell regulatory functions. J Immunol 178: 271-279, 2007.

48. Marin V, Montero-Julian FA, Gres S, Boulay V, Bongrand P, Farnarier C and Kaplanski G: The IL-6-soluble IL-6Ralpha autocrine loop of endothelial activation as an intermediate between acute and chronic inflammation: an experimental model involving thrombin. J Immunol 167: 3435-3442, 2001. 